$\xi=$

\title{
Neural oscillations under binocular rivalry: a brain activity pattern formation perspective
}

\author{
T. D. Frank ${ }^{1 *}$, P. I. Galenko ${ }^{1}$ \\ ${ }^{1}$ Department of Psychological Sciences, CESPA, University of Connecticut, Storrs, Connecticut, United States \\ *Corresponding author E-mail: till.frank@uconn.edu
}

\begin{abstract}
Binocular rivalry is a phenomenon in which individuals react to two overlaid images that are presented to both eyes of the individuals as if the two images were presented one after each other in time in a repetitive fashion. Recently, human brain activity has been measured during binocular rivalry. Previously reported brain activity data was evaluated and modeled from a human pattern formation perspective that states that the human brain in binocular rivalry experiments acts as a neural self-oscillator. The neural self-oscillator forms and dissolves brain activity patterns related to the two images in an alternating, rhythmic self-organized fashion. Task-related oscillatory components hidden in the brain activity raw data were identified using power spectral analysis. Those oscillatory components were subsequently modelled using a previously developed mathematical model for brain activity self-oscillations. The identified task-related oscillatory components showed similar power spectrum functions for both brain activity patterns. A reciprocal relationship between the amplitudes of the two patterns was found. The oscillator model was able to reproduce the power spectrum functions when noisy perturbations were taken into account.
\end{abstract}

Keywords: Binocular Rivalry; Neural Oscillations; Vision; Pattern Formation; Modeling.

\section{Introduction}

Human oscillatory brain dynamics induced by binocular rivalry, that is, by exposing in an appropriate way the two eyes of an individual to two different images that are overlaid to form one image, is an important phenomenon to obtain insights into human brain functioning (Pettigrew 2001, Leopold \& Logothetis 1999, Blake \& Logothetis 2002, Frank 2019, Hernandez-Lorca et al. 2019). In particular, binocular rivalry oscillations are a testbed for the general theoretical view of humans as self-organizing, living systems that form brain activity and body patterns (Haken 1996, Frank 2014a, Frank 2019). Binocular rivalry exploits a situation that can rarely be found in everyday life. Both eyes of an individual focus the same point. Nevertheless, the two eyes are exposed to different images. Various techniques can be used to establish such an experimental situation (Carmel et al. 2010). Healthy individuals subjected to such rivalry conditions typically react as if the two images were presented in an alternating sequence one after another (Blake \& Logothetis 2002, Leopold et al. 2002). Not only humans, but also monkeys react to binocular rivalry conditions in this way (Myerson et al. 1981). On the neural level, oscillatory brain activity can be observed both in humans (Tong et al. 1998, Hernandez-Lorca et al. 2019) and animals (Leopold \& Logothetis 1999). Therefore, it has been suggested that the human brain acts like a (self-)oscillator (Pettigrew 2001). This self-oscillator hypothesis has been proposed in the context of similar experimental visual paradigms that lead to oscillatory human reactions to unchanged external circumstances such as the Necker cube paradigm (Haken 1996), and the motion-induced blindness and spinning dancer paradigms (Frank 2014b, Frank \& O'Leary 2018, Frank 2019). The pattern formation perspective that states that humans are self-organizing, living systems that form brain activity and body patterns (Frank 2019) provides a theoretical "umbrella" framework that can address such paradigms in a unifying way. In what follows, human brain activity data from Tong et al. (1998) will be presented and analyzed within the aforementioned pattern formation perspective. In particular, a brain activity self-oscillator will be presented that mimics the observed experimental neural oscillations and shows how these neural oscillations may emerge as a result of short-term neuroplasticity of the human brain.

\section{Methods}

\subsection{Materials and data}

Data was taken from Tong et al. (1998). Tong et al. (1998) used functional magnetic resonance imaging (fMRI) to study brain activity induced by binocular rivalry images in certain areas of the human brain in a sample of $n=4$ participants. Tong et al. (1998) focused on two visual areas: human fusiform face area (FFA) which reacts to facial images and the parahippocampal place area (PPA) which reacts to non-facial images like houses and places. For a schematic indicating FFA and PPA locations see Fig, 1. Tong et al. (1998) used face/house rivalry images by having participants wear red and green filter glasses. Only one eye could see a face, and the other eye could only see a house. To induce binocular rivalry Tong et al. (1998) overlaid the green face percept with the red house creating a mixed im- 
age where binocular rivalry occurred. Participants were given a button box where they reported which kind of image (house versus face) became more dominant over time while a fMRI recorded their FFA and PPA areas. Tong et al. (1998) presented in their Figure 3 two 75s long fMRI signals from FFA and PPA of participant S1. The interval started at 105s after a fixation period (which was the second fixation period) and ended at 180s with another fixation period (which was the third fixation period). Figure 3 from Tong et al. (1998) was extracted and overlaid with a measurement grid so that the grid coincided with the range of the figure. The quantified data was then converted into a graph and overlaid on top of the original to check for accuracy.

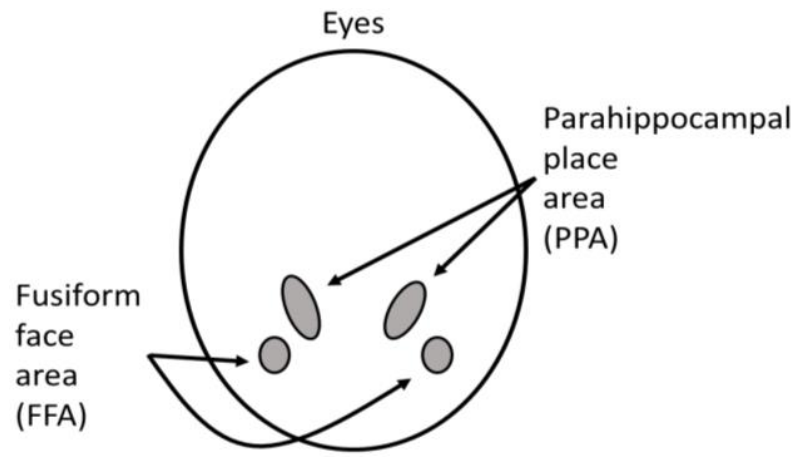

Fig. 1: Schematic showing the locations of the fusiform face area (FFA) and parahippocampal place area (PPA) in the left and right hemispheres of the human brain.

\subsection{Data analysis and modelling}

In the $75 \mathrm{~s}$ interval under consideration participant $\mathrm{S} 1$ saw 5 times the face image and 4 times the house image. The subinterval that spaned from the first time S1 saw a face to the fifth time when S1 saw a face was about 70s long. It contained 4 full oscillation periods under the assumption that each oscillation period comes with the emergence of 1 face-related and 1 house-related brain activity pattern. Therefore, the task-related oscillation period was about $70 \mathrm{~s} / 4=17.5 \mathrm{~s}$ such that the corresponding oscillation frequency was about $0.06 \mathrm{~Hz}$. In order to extract the task-related oscillatory components from the original FFA and PPA brain signals, a band-pass filter was applied to those signals with cut-off frequencies at $0.05 \mathrm{~Hz}$ (lower boundary) and $3.0 \mathrm{~Hz}$ (upper boundary). The power spectrum functions for both filtered signals were determined. In addition, moving-window averages with $0.07 \mathrm{~Hz}$ window width of the power spectrum functions were computed. Finally, inspired by a particular analysis method use by Tong et al. (1998) in which 20s long segments were averaged (for details see the conclusion section below), we cut the filtered $75 \mathrm{~s}$ long trajectories into 4 consecutive segments of $19 \mathrm{~s}$ length and averaged those segments.

In order to compare the experimental data with the brain activity self-oscillator model developed within the brain activity pattern formation perspective, the human model presented in Frank (2014b, 2019) was used. Accordingly, individuals performing in binocular rivalry experiments in general and the participant S1 in particular, were considered as human C2 systems (Frank 2019). Figure 2 shows the components of a human $\mathrm{C} 2$ system and the relevant model parameters. Following the $\mathrm{C} 2$ system schematic, we described $\mathrm{S} 1$ in terms of his or her brain state and brain structure. In the context of the rivalry experiment, the brain forms alternatively two brain activity patterns. One pattern located in the FFA, the other located in the PPA. In line with the theory of pattern formation, emerging brain activity patterns were described in terms of pattern amplitudes (Frank 2019). These amplitudes were denoted by $\mathrm{A}_{1}$ (FFA pattern) and $\mathrm{A}_{2}$ (PPA pattern), respectively. The structure was described in terms of the eigenvalues of the basis patterns underlying the FFA and PPA patterns (for more details about basis patterns, see Haken 1996 and Frank 2019). These eigenvalues were assumed to reflect connectivity weights of neuronal networks and were allowed to vary in time to some degree (neural plasticity). Importantly, following the notion of a human $\mathrm{C} 2$ system (Frank 2019), it was assumed that the brain state given in terms of the amplitudes $A_{1}$ and $A_{2}$ affected the structure given in terms of the eigenvalues $\lambda_{1}$ and $\lambda_{2}$, as indicated by the arrow that points from the Brain-State-box to the Structure-box. Vice versa, the structure ( $\lambda_{1}$ and $\lambda_{2}$ ) was assumed to determine the evolution of the brain state ( $A_{1}$ and $\left.A_{2}\right)$, as indicated by the arrow that points from the Structure-box to the Brain-State-box. Therefore, as discussed in detail in Frank (2019), the C2 system model under consideration featured a circular causality loop. A mathematical analysis (Frank 2014b, 2019) shows that under appropriate condition this loop can lead to brain activity oscillations in which the two brain activity patterns under consideration emerge in an alternating fashion. We used the $\mathrm{C} 2$ system model for those conditions. Finally, in general, a C2 system takes the impact of the environment into account. In the rivalry experiment by Tong et al. (1998), the environment was given in terms of the light that was reflected from the two images towards the participant S1. The C2 system approach takes a rigorous physics perspective according to which light is an electromagnetic force. Therefore, the box on the left is labelled "Force". The force was assumed to affect the brain state such that task-related brain activity patterns emerged, which is indicated by the arrow from the Force-box to the Brain-State-box.

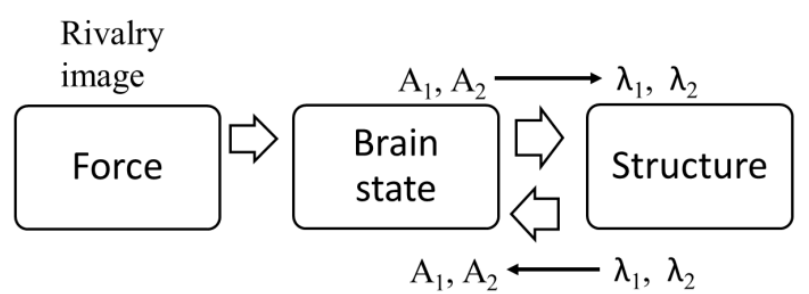

Fig. 2: Components and key variables of a human $C 2$ system applied to describe a participant performing in a binocular rivalry experiment. $A_{1}$ and $A_{2}$ are pattern amplitudes of the FFA And PPA brain activity patterns. $\lambda_{1}$ and $\lambda_{2}$ are eigenvalues that describe brain structure.

The evolution equations that determine how the state variables $A_{1}$ and $A_{2}$ and the structure variables $\lambda_{1}$ and $\lambda_{2}$ evolve in time were taken from Frank (2014b,2019). More precisely, Eqs. 7.10 and 7.11 from Frank (2019) for cubic nonlinearities were used and solved numerically. For those model equations it is known that the oscillation period $\mathrm{T}$ reads $\mathrm{T}=2 \tau \cdot \log (\mathrm{g} \cdot \mathrm{ms})$, where $\tau$ and $\mathrm{g}$ are model parameters and $\log (\mathrm{x})$ is the natural logarithm of $\mathrm{x}$ (Frank 2014b). We used the parameters $\tau=4 \mathrm{~s}$ and $\mathrm{g}=\exp (2) / \mathrm{ms}$ such that the period T was $16 \mathrm{~s}-\mathrm{simi}$ - 
lar to the aforementioned experimentally observed period. Other model parameters were (see Frank 2019 for details) $\gamma($ up $)=\gamma($ down $)=1 / \tau$, $\lambda 0=0, \lambda$ (baseline) $=\mathrm{g}+1 / \mathrm{ms}$ (as in Frank 2014b), $\theta=80 \%$. We determined the power spectrum functions of the time series $\mathrm{A}_{1}$ and $\mathrm{A}_{2}$ obtained from the simulations. Since the model is deterministic, we also created a version of the model that involved various noise terms that perturbed the model dynamics in a random fashion. Note that such noise terms are in fact also deterministic in nature: they reflect deterministic perturbations of various components of the human system or the environment that are not explicitly modelled (Frank 2019, Section 2.4).

\section{Results}

Figure 3 show the quantified data from participant S1 reported by Tong et al. (1998) and the results of the power spectral analysis and segment averaging analysis. Panel A shows the FFA (top subpanel) and PPA (bottom subpanel) signals as functions of time during a 75s duration that started after the second fixation period (see Section 2.1 above). Visual inspection shows that for both locations the taskrelated oscillatory signals with a period of about $17.5 \mathrm{~s}$ are hidden or obscured by ongoing, non-task-related brain activity with different oscillation frequencies. Panel B shows the power spectrum functions of the band-pass filtered signals shown in panel A without (thin lines) and with (thick lines) moving-window averaging. The top and bottom subpanels of panel B reveal that in the time series shown in panel A there are indeed oscillatory signals with frequencies around $0.06 \mathrm{~Hz}$. Those signals are assumed to be related to the participant's reactions of seeing a face or a house, respectively. The moving-window average functions (thick lines) show that the filtered FFA and PPA signals exhibit similar distributions of oscillatory frequencies. Finally, panel C presents the segment-averaged filtered data. Clearly, a reciprocal relationship between FFA and PPA activity as measured in terms of those filtered data can be seen.

A
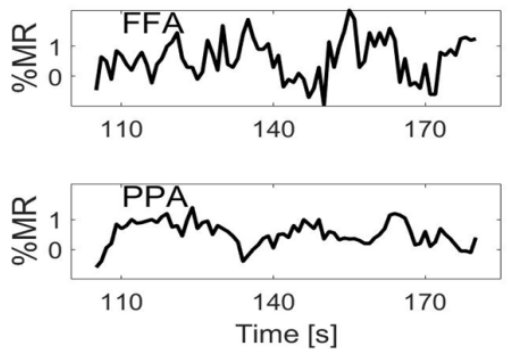

B
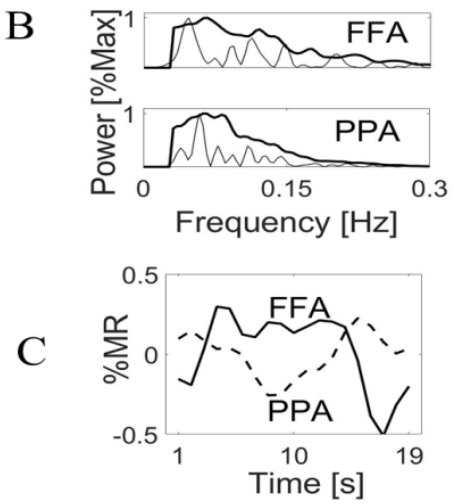

Fig. 3: FFA and PPA brain signals observed by Tong et al. (1998) for participant S1 (panel A) and the corresponding power spectrum functions (panel B) and filtered segment averages (panel C). In panel B the thin solid lines show the raw power spectrum functions, whereas the thick solid lines show the moving-window averages. In panel $\mathrm{C}$ the solid and dashed lines denote the filtered and 19s averaged FFA and PPA signals, respectively.

Figure 4 shows the results of the simulations of the human $\mathrm{C} 2$ system model sketched in Fig. 2. The top (A,B,C) and bottom (D, E, F) panels show simulation results for the deterministic and noisy models, respectively. Panel A depicts the amplitudes $\mathrm{A}_{1}$ and $\mathrm{A}_{2}$ of the brain activity patterns emerging in the FFA and PPA locations, respectively. Panel B describes the evolution of the brain structure characterized by the FFA and PPA eigenvalue variables $\lambda_{1}$ and $\lambda_{2}$. Taken together, panels A and B describe an alternating emergence of the FFA and PPA brain activity patterns (panel A) and an oscillatory variation of the underlying brain structure (panel B). Panel C presents the power spectral functions of the amplitude time series $A_{1}$ and $A_{2}$ shown in panel A. The two functions show a relative sharp main peak at a frequency of $0.06 \mathrm{~Hz}$ and smaller peaks at higher harmonics of $0.19 \mathrm{~Hz}$ (about 3 times of the main peak at $0.06 \mathrm{~Hz}$ ) and $0.31 \mathrm{~Hz}$ (about 5 times of the main peak at $0.06 \mathrm{~Hz}$ ). These high harmonics occur because the period functions $\mathrm{A}_{1}$ and $\mathrm{A}_{2}$ do not correspond to sine-functions but correspond to sequences of tooth-shaped bursts. Note that although the main peaks at $0.06 \mathrm{~Hz}$ are relatively narrow (i.e., have a relatively small bandwidth), they do not correspond to line-like peaks, as one may expect from a deterministic model. We will return to this issue in the conclusion section. Finally, the power spectral functions of $A_{1}$ and $A_{2}$ are identical (at least on the scale shown in panel $\mathrm{C}$ ). The reason for this is that the functions $\mathrm{A}_{1}$ and $\mathrm{A}_{2}$ are identical except for a shift in time. As far as the noisy model (see Section 2.2. for details) is concerned, comparing panels D, E, F with panels A, B, C we can point out communalities and differences. Comparing panels $\mathrm{A}$ and $\mathrm{D}$, we see that the noisy model produces small variations in the durations of the epochs that describe a particular pattern emerging and dominating over the other pattern for a while. However, these variations are relatively small. Suggestions about how to increase the variability of those durations in order to increase the fit of the model and experimental data will be made in the conclusion section. Comparing panels D and $\mathrm{E}$ with $\mathrm{A}$ and $\mathrm{B}$, we see that the brain state and brain structure time series produced by the noisy model exhibit jitter, as expected. Comparing the thin solid lines in panel $\mathrm{F}$ with the thick solid lines in panel $\mathrm{C}$, we see that the power spectrum functions of the noisy model are wider. Moreover, a close look at the thin-lined power spectrum functions for FFA and PPA in panel F shows that they are no longer identical. The reason for this is that the random elements introduced in the noisy model act independently on the two brain locations. Finally, for the noisy model the moving-window power spectrum functions shown in solid thick lines in panel $\mathrm{F}$ resemble to some extent the moving-window power spectrum functions obtained from the experimental data as shown in panel B of Fig. 3. 
A
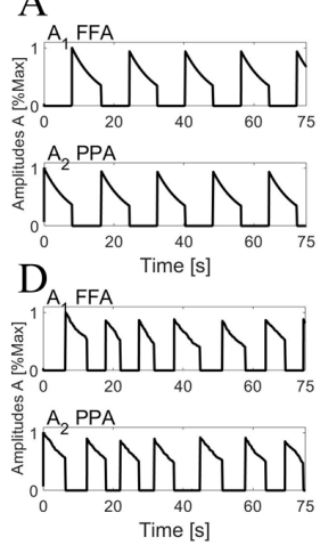

B
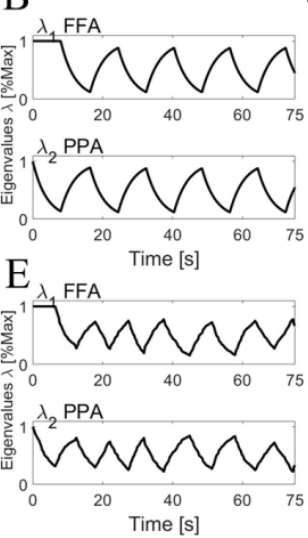

$\mathrm{C}$

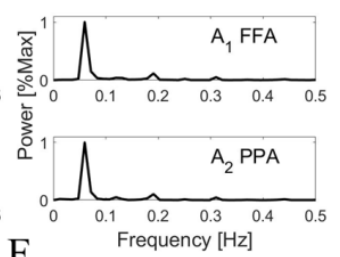

$\mathrm{F}$

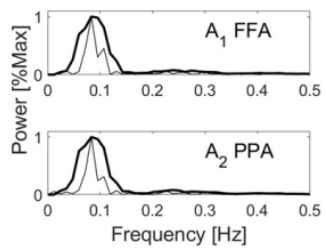

Fig. 4: Simulation results of the brain activity self-oscillation model suggested in Frank (2014b, 2019) describing mathematically the human C2 system sketched in Fig. 2. Panels A, B, C and D, E, F describe the model performance without and with noise added to the model, respectively. Panels A, D: Pattern amplitudes $\mathrm{A}_{1}$ and $\mathrm{A}_{2}$ related to the brain activity patterns emerging in the FFA and PPA locations. Panels B, E: Eigenvalues $\lambda_{1}$ and $\lambda_{2}$ describing oscillatory variations of brain structure. Panels $\mathrm{C}, \mathrm{F}$ : Power spectrum functions of $\mathrm{A}_{1}$ and $\mathrm{A}_{2}$. Thick lines in panel $\mathrm{C}$ and thin lines in panel $\mathrm{F}$ are the power spectrum functions without moving-window averaging. Thick lines in panel $\mathrm{F}$ describe the moving-window averages of the original (thin lines) power spectrum functions.

\section{Conclusions}

We extracted, re-analysed, and modelled brain activity data record by Tong et al. (1998) in a binocular rivalry experiment. The data was given in terms of two time series that described brain activity from two presumably interacting but (in some sense) mutually exclusive brain activity patterns. Using power spectral analysis and band-pass filters we were able to identify task-related oscillatory components of the original time series. We showed that those oscillatory components of the two patterns satisfied a reciprocal relationship. When one component was large, the other component was small, and vice versa. A model proposed earlier within the framework that addresses humans as pattern formation systems was applied to describe the self-oscillatory dynamics of the amplitudes of the two brain activity patterns. The model replicated the task-related oscillatory components on the level of their power spectrum functions.

Our analysis revealed that the brain activity in the two talk-related locations, FFA and PPA, did not show a clear periodic temporal pattern. The reason for this is at least twofold. First of all, it is plausible to assume that at the cortical areas of interest in addition to the taskrelated brain activity there is non-task-related, ongoing brain activity. This non-task-related brain activity obscures the task-related temporal pattern. Second, the epochs during which a brain activity pattern dominates are known to vary in their durations. Typically, the durations are Gamma distributed (Leopold \& Logothetis 1996, Tong et al. 1998). In order to cope with the first issue, Tong et al. extracted 20s intervals centred around the manual reactions of their participants and averaged those intervals. They found a reciprocal relationship that when brain activity in one of the two locations was relatively high, then the activity was low in the other region. In contrast, in our study, the task-related periodic neural components of the FFA and PPA signals were extracted by applying a band-pass filter to the signals. When considering moving-window averages, then FFA and PPA signals showed a similar distribution of oscillatory frequencies. Moreover, when averaging 19s intervals of the filtered signals, we observed a reciprocal relationship between FFA and PPA filtered signals similar to that reported by Tong et al. (1998).

The brain activity self-oscillator model that was suggested in the literature within the framework of humans as self-organizing, living pattern formation systems (Frank 2014b,2019) was able to reproduce the putative task-related brain activity oscillations at frequencies of about $0.06 \mathrm{~Hz}$. Although the model is a deterministic model, it did not produce a peak in terms of a line at a particular frequency. The reason for this is the finiteness of the simulated time series. We repeated the simulations using a three times longer interval (i.e., a $225 \mathrm{~s}$ interval) and found that the power spectrum functions obtained from the simulated times series $\mathrm{A}_{1}$ and $\mathrm{A}_{2}$ showed sharp, line-like peaks. Moreover, those peaks were at the theoretically predicted frequency of $1 / 16 \mathrm{~s}=0.062 \mathrm{~Hz}$. Recall that for the $75 \mathrm{~s}$ interval simulations the obtained peaks were found to be at about $0.060 \mathrm{~Hz}$ (see Section 3). That is, in general, the model produced oscillation frequencies at or close to the theoretically predicted value. The reason for this is that the predictive formula $\mathrm{T}=2 \tau \cdot \log (\mathrm{g} \cdot \mathrm{ms})$ holds under the assumption that the time constant of the brain state dynamics is much smaller than the time constant of the structure dynamics (Frank 2014b). That is, the structure dynamics is slow. The time constant of the structure dynamics is $\tau$. An approximative measure for the time constant of the state (or amplitude) dynamics is given by the parameter $1 / \lambda$ (baseline). In our simulation we had $\tau=4000 \mathrm{~ms}$ and $1 / \lambda($ baseline) $=0.1 \mathrm{~ms}$. Clearly, the time constant of the structure dynamics was much larger. The structure was the slowly evolving component.

In order to obtain a simulation output that mimics the relative broad power spectrum functions that we derived from the human data by Tong et al. (1998), we added random elements to the deterministic brain oscillator model. As expected, the power spectrum functions of the noisy oscillator model showed a broader distribution of frequencies. Therefore, the noisy oscillator model may be considered as a more realistic model of a human individual performing in a binocular rivalry experiment. However, the amplitudes time series $\mathrm{A}_{1}$ and $\mathrm{A}_{2}$ showed even in the noisy model very regular, periodic patterns, see Fig. 4. In particular, it seemed that the noise had a small effect on the epoch durations during which one of the two amplitudes was "on", while the other was "off". Therefore, future studies may be devoted to create a noisy oscillator model that reproduces the aforementioned Gamma distributed epoch durations. The simplest realisation of such a model is to put Gamma distributed parameters into the model. In the context of the simulations presented above, we would define a baseline time interval of $16 \mathrm{~s}$. After every $16 \mathrm{~s}$ period, we would choose an epoch duration $\mathrm{T}$ from a Gamma distribution. Given the randomly obtained value for $\mathrm{T}$, we would, for example, adjust the time constant $\tau$ like $\tau=\mathrm{T} /(2 \log (\mathrm{g}))$, where $\mathrm{g}$ would be fixed for the whole simulation period. This example illustrates that the current study opens an avenue for future studies that focus, on the one hand, on the modelbased analysis of brain activity data from binocular rivalry experiments, or, on the other hand, on modelling such data by means of brain activity self-oscillators and within the perspective of humans as pattern formation systems. 


\section{References}

[1] Pettigrew JD (2006), searching for the switch: neural basis for perceptual rivalry alternations. Brain and Mind 2, 85-118. https://doi.org/10.1023/A:1017929617197.

[2] Leopold DA \& Logothetis NK (1999), Multistable phenomena: changing views in perception. Trends in Cognitive Sciences 3, 254-264. https://doi.org/10.1016/S1364-6613(99)01332-7.

[3] Blake R \& Logothetis NK (2002), Visual competition. Nature Reviews Neuroscience 3, 13-31. https://doi.org/10.1038/nrn701.

[4] Hernandez-Lorca M, Sandberg K, Kessel D, Fernandez-Folgueiras U, Overgaard M \& Carretie L (2019), Binocular rivalry and emotion: Implications for neural correlates of consciousness and emotional biases in conscious perception. Cortex 120, 539-555. https://doi.org/10.1016/j.cortex.2019.08.003

[5] Haken H (1996), Principles of brain functioning. Springer, Berlin. https://doi.org/10.1007/978-3-642-79570-1.

[6] Frank TD (2014a), A nonlinear physics model based on extended synergetics for the flow of infant actions during infant-mother face-to-face communication. International Journal of Scientific World 2, 62-74. https://doi.org/10.14419/ijsw.v2i2.3468.

[7] Frank T (2019), Determinism and self-organization of human perception and performance. Springer, Berlin. https://doi.org/10.1007/978-3-03028821-1

[8] Carmel D, Acraro M, Kastner S \& Hasson U (2010), How to create and use binocular rivalry. Journal of Visualized Experiments 45, e2030. https://doi.org/10.3791/2030.

[9] Leopold DA, Wilke M, Maier A \& Logothetis NK (2002), Stable perception of visually ambiguous patterns. Nature Neuroscience 5, 605-609. https://doi.org/10.1038/nn0602-851.

[10] Myerson J, Miezin F \& Allman J (1981), Binocular rivalry in Macaque monkeys and humans: a comparative study in perception. Behaviour Analysis Letters 1, 149-159.

[11] Tong F, Nakayama K, Vaughan JT \& Kanwisher N (2007), Binocular rivalry and visual awareness in human extrastriate cortex. Neuron 21, 753759. https://doi.org/10.1016/S0896-6273(00)80592-9.

[12] Frank TD (2014b), Multistable perception in schizophrenia: a model-based analysis via coarse-grained order parameter dynamics and a comment on the 4.th law. Universal Journal of Psychology 2, 231-240.

[13] Frank TD \& O’Leary J (2018), Motion-induced blindness and the spinning dancer paradigm: a neuronal network approach based on synergetics. In Horizon in Neuroscience Research (Costa A \& Villalbaon E eds.), Nova Publishers, New York, pp. 51-80. 